Original Article

\title{
FORMULATION AND OPTIMIZATION OF TERBINAFINE HCI SOLID LIPID NANOPARTICLES FOR TOPICAL ANTIFUNGAL ACTIVITY
}

\author{
FATMA E. ABOBAKR ${ }^{1}{ }^{*}$, SAHAR M. FAYEZ², VIVIAN S. ELWAZZAN1, WEDAD SAKRAN³
}

${ }^{1}$ National Organization for Drug Control and Research (NODCAR), ${ }^{2}$ Faculty of Pharmacy, October $6^{\text {th }}$ University, ${ }^{3}$ Faculty of Pharmacy, Helwan University

Email: fatma_abobakr89@yahoo.com

Received: 03 Sep 2019, Revised and Accepted: 30 Oct 2019

\begin{abstract}
Objective: Solid lipid nanoparticles (SLNs) are at the forefront of the rapidly developing field of nanotechnology with several potential applications in drug delivery and research. The aim of this study was to develop and characterize SLNs formulae of Terbinafine HCl (TFH) for topical drug delivery applications.
\end{abstract}

Methods: SLNs were prepared using the solvent injection technique. Glyceryl Monostearate (GMS) served as the lipid base. Three stabilizers; Tween 80, Cremophor RH40, and Poloxamer 188, were used. The effect of stabilizer type and concentration, as well as the lipid concentration, were studied, factorial design of $3^{2 *} 2^{1}$ was applied. The prepared SLNs were characterized regarding their particle size, zeta potential, polydispersity index (PDI), entrapment efficiency percent (EE \%), and physicochemical stability. The selected formulae were subjected to further investigations such as morphological studies, in vitro release studies, and Infrared (IR) spectroscopy. They were compared with the marketed cream Lamifen ${ }^{\circledR}$ in term of their antifungal activity against Candida albicans.

Results: Lipid concentration, together with the type and concentration of stabilizer, appeared to be the main cornerstones which affect the formation of SLNs. Smaller particle size was observed when increasing the stabilizer concentration and decreasing the lipid concentration. Higher EE\% was observed when increasing both the stabilizer and the lipid concentrations. Formulae (F6, F12 andF19) were selected as the most suitable SLNs with optimum particle size of $480.2 \pm 18.89,458.6 \pm 12.45$ and $246.7 \pm 10.5 \mathrm{~nm}$, respectively as well as the highest EE $\%$ of $87.13 \pm 0.19,93.69 \pm 0.7$ and $95.06 \pm 0.25$, respectively. In vitro microbiological screening of their antifungal activity showed significantly larger zones of inhibition of diameters $25.9 \pm 0.25,25 \pm 0.35$ and $24.67 \pm 0.36 \mathrm{~mm}$, respectively in comparison with the marketed Lamifen ${ }^{\circ}$ cream which showed a zone of $11.2 \pm 0.44 \mathrm{~mm}$ diameter.

Conclusion: Applying SLNs containing TFH as topical antifungal preparations may be considered as a very promising option as they show good physicochemical characterization with high antifungal activity, which delineates them as a promising dosage form for topical antifungal treatment.

Keywords: Solid lipid nanoparticles (SLNs), Solvent injection technique, Terbinafine HCl (TFH), Topical drug delivery

(C) 2019 The Authors. Published by Innovare Academic Sciences Pvt Ltd. This is an open access article under the CC BY license (http://creativecommons.org/licenses/by/4.0/) DOI: http://dx.doi.org/10.22159/ijpps.2019v11i12.35560

\section{INTRODUCTION}

Nanoparticles (NPs) are colloidal drug carriers that range between 1 to 1000 nanometres $(\mathrm{nm})$ in size with a surrounding interfacial layer. NPs have many advantages such as low cost of ingredients, ease of preparation as well as being suitable for the preparation of hydrophilic and lipophilic drugs. These particles also designed to enhance the solubility of poorly soluble drugs [1-3]. NPs may be classified according to the type of polymer used in their preparation, and, so, they are called polymeric NPs or according to the type of lipid used and, hence, called lipid-based NPs. Polymeric NPs are colloidal carriers composed of biodegradable macromolecular polymers. They increase the therapeutic performance of the poorly soluble drug in any route of administration, but unfortunately, they suffer from toxic degradation of toxic monomers aggregation [4]. Lipid-based colloidal particles have an advantage over polymeric systems. They avoid the toxic effect, which may be obtained by the polymers used in the polymeric system $[5,6]$.

Lipid NPs can be classified based on the type of the lipid used. Many lipids can be used, such as fatty acids, glycerides, and waxes. Some important factors must be considered during the selection of the lipid phase, such as the solubility of the drug in it, the partitioning of the drug between the lipid and aqueous phase, and the lipid polymorphism. A solid lipid was selected in the study to produce solid lipid nanoparticles (SLNs). Using solid lipids in the formulation of NPs has an advantage of reducing drug mobility, decreasing drug leakage and also decreases its migration into the interfacial layer. SLNs also have the advantage of improving the bioavailability of the drug by increasing their dissolution and absorption [4].
The main components of SLNs are solid lipid, stabilizers, and active ingredients. Co-stabilizers may be used. Stabilizers are another name for the surface active agents or the emulsifier. They are the key of formation and stabilization of lipid NPs. They help in dispersing the lipid in the aqueous phase during the manufacturing process. The main action of stabilizer is lowering the surface free energy; thus, the surface tension between the two phases will decrease (first role). Once the lipid NPs were formed, the stabilizer performs its second function of stabilizing its structure (second role) [7].

Terbinafine $\mathrm{HCl}$ (TFH) is effective in the treatment of different skin diseases such as dermatophytosis, candidiasis, seborrhoea dermatitis, and onychomycosis. Topical therapy is usually the most preferred due to the ease of administration. SLNs possess several advantages for the topical route of administrations as they increase the penetration of the encapsulated drug into the skin along with the avoidance of the first-pass metabolism, the avoidance of side effects as allergic reactions, and also targeting the active ingredient to the affected areas over the skin $[8,9]$.

The aim of this study was to develop and characterize SLNs formulae of TFH for topical drug delivery system.

\section{MATERIALS AND METHODS}

\section{Materials}

Terbinafine $\mathrm{HCl}$ (TFH); kindly gifted by Amoun Pharmaceutical Company, Egypt. Glyceryl monostearate (GMS); BDH Chemicals, Ltd Poole England. Poloxamer 188 and cremophor RH40; FlukaChemie, Sigma Aldrich, ChemieGmblt, Switzer land. Ethanol and Tween 80; 
Adwic, El-Nasr Pharmaceutical Chemical Company, Egypt. All chemicals are of analytical grade and used without further purification. The fungal strain Candida albicans (strain ATTC 10231) was obtained from National Research Center, Cairo, Egypt. Sonicator (type USR3) (JulaboLabortechnik, Seelbach, West Germany). Hot plate with a magnetic stirrer (Model300HS, Japan). Centrifuge: Sigma, Germany. IR Spectrophotometer (IR-470) (Shimadzu, Kyoto, Japan). Transmission Electron Microscope (TEM) (JEOL, JEM-2100, Japan). Sensitive Electric Balance (Shimadzu, Libror AEG-220, Japan). UV-visible spectrophotometer (model 1800, Shimadzu Tokyo, Japan). Zetasizer Nano (model ZS3600, Malvern Instruments Ltd., Worcestershire, UK).

\section{Methodology}

\section{Preparation of SLNs of TFH}

In this study, the solvent injection technique was the method used to prepare TFH SLNs. The solid lipid was dissolved in a water-miscible solvent (e. g., ethanol, methanol, and acetone) or in a mixture of these solvents together. This lipid solvent mixture was injected through an injection needle into a stirred aqueous phase with or without a stabilizer. The presence of a stabilizer within the aqueous phase helped to produce lipid droplets at the site of injection and stabilize SLNs by reducing the surface tension between water and solvent $[10,11]$.

TFH (1\%) was mixed with glyceryl monostearate (GMS) and then dissolved into ethanol. They were heated together to above the melting point of the lipid $\left(>55^{\circ} \mathrm{C}\right)$. The resulted organic solution was injected at once into the aqueous phase (distilled water and the nonionic stabilizer). The aqueous phase immediately turned milky, and this indicated the formation of lipid NPs suspension. The resulted suspension was stirred at $500 \mathrm{rpm}$ for three hours on a magnetic stirrer. After that, SLNs suspension was sonicated for $15 \mathrm{~min}$.

\section{Factorial design}

The factorial design was considered as an efficient statistical tool to study the influence of numerous factors concurrently. In addition, it was more practical to predict and estimate the interaction between selected variables and thus reduced the number of experimental runs. Stepwise multivariable regression analysis was also used to determine the significant factors.

Eighteen formulae were prepared and entered in JMP (version 14 software SAF instate inc., Cary NC), a factorial design program used to select the optimized formula. A $3^{2 *} 2^{1}$ full factorial design was used to define the optimum conditions regarding the selected factors. The design involved two factors, each with three levels and one factor with two levels. The two factors with the three levels reflect the concentration of GMS $(2.5,5$ and $10 \%)$ and the stabilizer types (Tween 80, Cremophor RH40 and Poloxamer 188) and the one factor with the two levels reflect the stabilizer concentration ( 1 and $2 \%$ ) as shown in table 1 .

Table 1: Composition of the various formulae of TFH SLNs

\begin{tabular}{|c|c|c|c|c|c|}
\hline \multirow[t]{2}{*}{ Formula code } & \multirow[t]{2}{*}{ TFH (mg) } & \multirow[t]{2}{*}{ GMS (\%) } & \multicolumn{3}{|c|}{ Stabilizer (\%) in the aqueous phase } \\
\hline & & & Tween 80 & Cremophor RH40 & Poloxamer 188 \\
\hline F1 & 100 & 2.5 & 1 & & \\
\hline $\mathrm{F} 2$ & 100 & 2.5 & 2 & & \\
\hline F3 & 100 & 5 & 1 & & \\
\hline F4 & 100 & 5 & 2 & & \\
\hline F5 & 100 & 10 & 1 & & \\
\hline F6 & 100 & 10 & 2 & & \\
\hline F7 & 100 & 2.5 & & 1 & \\
\hline F8 & 100 & 2.5 & & 2 & \\
\hline F9 & 100 & 5 & & 1 & \\
\hline F10 & 100 & 5 & & 2 & \\
\hline F11 & 100 & 10 & & 1 & \\
\hline F12 & 100 & 10 & & 2 & \\
\hline F13 & 100 & 2.5 & & & 1 \\
\hline F14 & 100 & 2.5 & & & 2 \\
\hline F15 & 100 & 5 & & & 1 \\
\hline F16 & 100 & 5 & & & 2 \\
\hline F17 & 100 & 10 & & & 1 \\
\hline F18 & 100 & 10 & & & 2 \\
\hline
\end{tabular}

Table 2: Composition of the optimized formula based on $3^{2 *} 2^{1}$ factorial design

\begin{tabular}{llll}
\hline Formula code & TFH (mg) & GMS (\%) & \multicolumn{2}{l}{ Stabilizer (\%) in the aqueous phase } \\
\cline { 2 - 3 } & & Tween 80 & Cremophor RH40 \\
\hline F19 & 100 & 6.25 & 1.5 \\
\hline
\end{tabular}

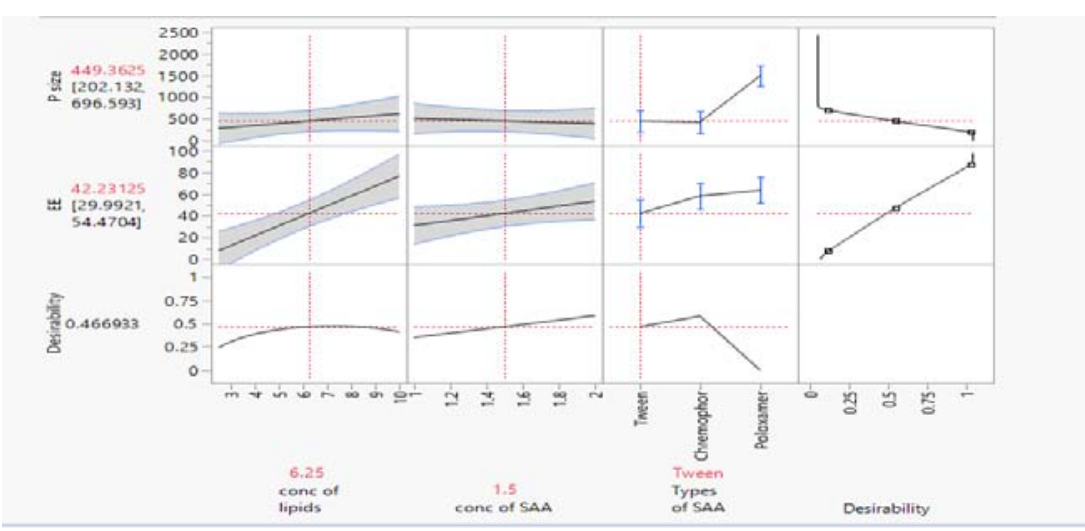

Fig. 1: Composition and characterization of the optimized formula (F19) 


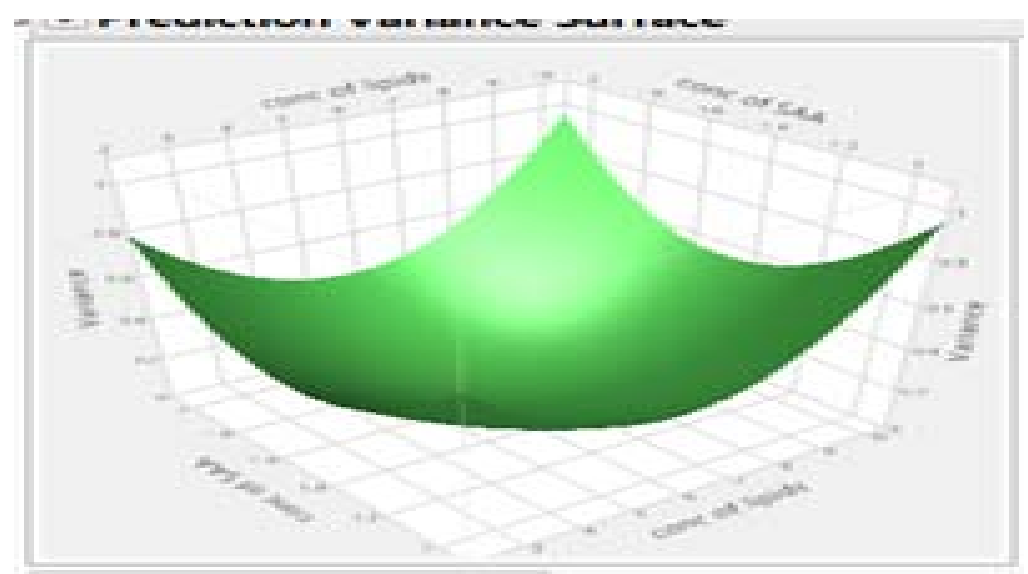

Fig. 2: Surface plot of the most desirable formula (F19)

\section{Selection of the optimized formula by factorial design}

Applying the desirability function of the used software gave rise to a new formula F19 with the following composition: $6.25 \%$ GMS and $1.5 \%$ tween 80 . This formula was the most optimized formula in the model (table 2). Fig. 1 illustrates the composition of the optimized formula F19 as well as its expected values of particle size and EE\%.

\section{Physicochemical characterization of TFH SLNs}

Particle size analysis, polydispersity index, and zeta potential measurement

Particle size analysis, polydispersity index (PDI), and zeta potential (ZP) were performed for all TFH SLNs formulae using Malvern Zetasizer. Prior to measurement, two drops of SLNs suspension were diluted in $10 \mathrm{ml}$ of distilled water and sonicated for $10 \mathrm{~min}$. After that, $0.1 \mathrm{ml}$ was taken and further diluted in another $10 \mathrm{ml}$ of distilled water and sonicated for $10 \mathrm{~min}$. The second diluted sample was filled in a transparent sizing cuvette $[12,13,6]$. The PDI value gave an indication of the homogeneity of the preparations [14]. All measurements were performed in triplicate.

\section{Determination of the drug entrapment efficiency percent (EE \%)}

The EE\% gave an indication about the suitability of the drug to be formulated in a SLNs system. EE\% was determined by the indirect method by measuring the concentration of the remaining free drug in the aqueous phase, which contained the SLNs dispersion by using the centrifugation method at $5000 \mathrm{rpm}$ for $30 \mathrm{~min}$ at $25^{\circ} \mathrm{C}$ [15]. The concentration of the free TFH was measured spectrophotometrically at $\lambda \max =283 \mathrm{~nm}$ [16]. All experiments were run in triplicates. EE \% was determined using the following equation:

$$
E E \%=\frac{W \text { initial drug }-W \text { free drug }}{W \text { initial drug }} \times 100
$$

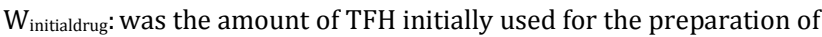
the SLNs,

$\mathrm{W}_{\text {free }}$ drug in suspension: was the amount of free drug determined in the aqueous phase after separation of the NPs [17].

\section{Morphology of TFH SLNs}

The morphology of the selected TFH SLNs suspension was observed using a transmission electron microscope (TEM). One drop of the diluted suspension sample was deposited on a film-coated 200-mesh copper grid, stained with one drop of $3 \%(\mathrm{w} / \mathrm{v})$ aqueous solution of phosphotungstic acid and allowed to dry. Any excess fluid was removed with filter paper before the examination [18].

\section{In vitro drug release study}

One gram of the optimized formulae (equivalent to $10 \mathrm{mg}$ of TFH) was placed in cellulose membrane, pore size $(0.45 \mu \mathrm{m})$, at the dissolution vessel of the USP dissolution test apparatus II. The dissolution media was $250 \mathrm{ml}$ of phosphate buffer ( $\mathrm{pH}$ 5.5) containing $0.8 \%$ tween 80 , which was added into the dissolution media to maintain the sink conditions. The release study was carried out at $37^{\circ} \mathrm{C} \pm 0.5$ [9]. The stirring paddle was rotated at a speed of 50 rpm [19]. Two ml samples were withdrawn and replaced by $2 \mathrm{ml}$ fresh phosphate buffer solution at 5, 10, 15, 30, 60, 90, 120, and 150 min time intervals. They were spectrophotometrically analyzed for TFH at its $\lambda \max 283 \mathrm{~nm}$. The blank was prepared according to the same procedure by using a plain SLNs suspension. The cumulative amount of TFH released was plotted as the function of time, and the release rate was calculated from the slope of the straight-line portion. Experiments were repeated three times as the results were expressed as the mean values \pm SD.

\section{Infrared (IR) spectroscopy}

Infrared spectra of physical mixtures of pure TFH with each one of the used excipients, namely GMS, Tween 80 and Cremophor40, as well as those of the selected optimum formulae, were investigated using $\mathrm{KBr}$ discs. The IR spectra were obtained in the spectral region $4000-400 \mathrm{~cm}^{-1}[20]$.

\section{Microbiological evaluation of the selected SLNs formulae}

The cup-plate technique was used to evaluate the antifungal activity of the selected SLNs, as described by Barot et al., and Sahoo et al., [21, 22]. The evaluation was carried out against Candida albicans ATCC 10231, which obtained from The National Research Centre, Cairo, Egypt. The selected formulae and the marketed lamifen ${ }^{\circledR}$ cream were prepared at a concentration of $1 \%$ TFH. Sabouraud's dextrose agar was used for the evaluation of these formulae against Candida albicans. Dextrose agar was poured into a sterile petri dish followed with surface inoculation of Candida albicans $\left(10^{7} \mathrm{CFU} / \mathrm{ml}\right)$. Three wells $(7 \mathrm{~mm}$ diameter hole cut) were made in the middle of the agar petri dish and filled with $0.1 \mathrm{ml}$ of the selected SLNs formulae, their plain base and lamifen ${ }^{\circledR}$ cream, respectively. The plate was then incubated at 37 ${ }^{\circ} \mathrm{C}$ for $48 \mathrm{~h}$. The diameter of zone of inhibition was measured in $\mathrm{mm}$ after incubation, recorded and considered as an indication for antifungal activity. This method was done in triplicate and mean results were calculated [23-25].

\section{Effect of the storage time on stability of the selected formulae}

TFH loaded SLNs optimized formulae were investigated for their stability. The samples were stored at controlled ambient temperature $\left(25^{\circ} \mathrm{C}\right)$ for three and six months. The samples were analyzed for their particle size, zeta potential, PDI and EE\%.

\section{Statistical analysis}

All data were expressed as mean \pm standard deviation $(n=3)$. They were subjected to one way ANOVA followed by Tukey Kramer's post-test using Instat 3 software. A difference between means was considered significant if the P-value was less than or equal to 0.05 . 


\section{RESULTS AND DISCUSSION}

\section{Physicochemical characterization}

The effect of different formulae variables and their interaction was studied and statistically analyzed. The results are listed in table 3 and illustrated in fig. 3.

\section{Particle size analysis and polydispersity index (PDI)}

Particle size was selected to be one of the dependent variables, and the PDI value gave an indication for the homogeneity of the preparations. PDI of all the prepared formulae was found to be (0.375-1).

\section{The effect of stabilizer type on the particle size}

The particle size of the prepared SLNs was significantly affected by changing the stabilizer type as follows, using poloxamer as stabilizer significantly increased the particle size $\left({ }^{* *} p<0.0001\right)$ while using any of Tween 80 or CremophorRH40 exerted a less significant negative effect and decreased the particle size of the SLNs $\left({ }^{*} \mathrm{p}<0.05\right)$. The SLNs formulae prepared using 1 and $2 \% \mathrm{w} / \mathrm{w}$ poloxamer 188 (F13 to F18) showed larger particle size than those prepared using $1 \%$ and $2 \% \mathrm{w} / \mathrm{w}$ tween 80 and cremophor RH40 (F1 to F12) (poloxamer $188<$ tween $80<$ cremophor RH40). This may be due to the higher molecular weight of poloxamer 188 compared to tween 80 and cremophor RH 40 [26]. Vitorino et al.[15] stated that the choice of stabilizer is important in the optimization of any NPs formulae. The authors added this component contributes not only to control the particle size and stability of the dispersions, but also to control the crystallization behavior, including polymorphs.

\section{The effect of stabilizer concentration on the particle size}

Changing the stabilizer concentration from 1 to $2 \%$ exerted a significant effect on particle size $\left({ }^{* *} \mathrm{p}<0.001\right)$. The average diameters of F7 (1\% cremophor RH 40) were $334.80 \pm 11.21 \mathrm{~nm}$ compared to F8 (2\% cremophor RH 40) $101 \pm 9.45 \mathrm{~nm}$. This was attributed to higher stabilizer concentration, which prevented particles' aggregation [27]. These results were in agreement with Tiwari S et al., who observed that the stabilizer concentration affects the particles' diameter. They attributed this to the increase in stabilizer concentration, which led to the reduction of the interfacial tension between the lipid matrix and the aqueous phase resulting in a formation of SLNs with smaller particle size $[28,29]$.

\section{The effect of lipid concentration on the particle size}

Changing in lipid concentration exerted a significant main effect on the particle size of the prepared SLNs $\left({ }^{*} \mathrm{p}<0.05\right)$. The particle size of the SLNs formulae prepared using higher lipid concentrations of $10 \%$ (F5, F6, F11, F12, F17, F18) was significantly larger $\left({ }^{*} \mathrm{P}<0.05\right)$ than their corresponding ones prepared using lower lipid concentration of $2.5 \%$ (F1, F2, F7, F8, F13, and F14), For example, the average diameter of F1 (2.5\% GMS) was $243.40 \pm 14.73 \mathrm{~nm}$ while that of F5 $(10 \% \mathrm{GMS})$ was $694.10 \pm 18.82 \mathrm{~nm}$. Moreover, the average diameter of F7 $(2.5 \% \mathrm{GMS})$ was $334.80 \pm 11.21 \mathrm{~nm}$ compared to F11 (10\% GMS) with $712.30 \pm 18.89 \mathrm{~nm}$ average diameter. According to Shahi and Pathak et. al., the change in particle size may be due to the tendency of lipid to coalesce at high lipid concentration [30,31]. The authors added that the lipid concentration was the most important factor affecting particles' diameter. They showed that increasing the lipid content of geleol, compritol 888 ATO and percirol ATO from 5 to 7.5 , and $10 \%$ resulted in a subsequent increase in particle size [32].

\section{Two-factor interaction}

The factorial design analysis revealed that there was a significant two-factor interaction effect of lipid concentration and type of stabilizer on the particle size of the prepared SLNs $\left({ }^{*} \mathrm{p}<0.05\right)$.

Table 3: The particle size, polydispersity index, zeta potential and entrapment efficiency of the investigated TFH loaded SLNs

\begin{tabular}{|c|c|c|c|c|}
\hline Formula code & ${ }^{*}$ Particle size $(\mathrm{nm})$ & *PDI & ${ }^{*} \mathbf{Z P}(\mathrm{mV})$ & *EE\% \\
\hline F1 & $243.4 \pm 14.73$ & $0.423 \pm 0.03$ & $-22.8 \pm 0.25$ & $3.30 \pm 0.03$ \\
\hline $\mathrm{F} 2$ & $195.5 \pm 18.51$ & $0.473 \pm 0.04$ & $-19.46 \pm 0.29$ & $7.33 \pm 0.08$ \\
\hline F3 & $500.7 \pm 12.45$ & $0.56 \pm 0.02$ & $-22.33 \pm 0.36$ & $14.21 \pm 0.05$ \\
\hline $\mathrm{F} 4$ & $470.3 \pm 10.15$ & $0.63 \pm 0.06$ & $-24.13 \pm 0.34$ & $54.68 \pm 0.1$ \\
\hline F5 & $694.1 \pm 11.21$ & $0.643 \pm 0.05$ & $-22.1 \pm 0.26$ & $63.77 \pm 0.16$ \\
\hline F6 & $480.2 \pm 18.89$ & $0.533 \pm 0.08$ & $-21.60 \pm 0.45$ & $87.13 \pm 0.19$ \\
\hline F7 & $334.8 \pm 11.45$ & $0.502 \pm 0.05$ & $-12.10 \pm 0.36$ & $4.69 \pm 0.3$ \\
\hline F8 & $101 \pm 9.45$ & $0.357 \pm 0.04$ & $-10.50 \pm 0.27$ & $19.75 \pm 0.5$ \\
\hline F9 & $478 \pm 12.65$ & $0.674 \pm 0.06$ & $-24.43 \pm 0.35$ & $51.79 \pm 0.2$ \\
\hline F10 & $371.6 \pm 11.18$ & $0.579 \pm 0.07$ & $-11.90 \pm 0.45$ & $65.39 \pm 0.16$ \\
\hline F11 & $712.3 \pm 15.18$ & $0.922 \pm 0.05$ & $-21.30 \pm 0.64$ & $89.63 \pm 0.14$ \\
\hline F12 & $458.6 \pm 12.45$ & $0.477 \pm 0.07$ & $-21.80 \pm 0.39$ & $93.69 \pm 0.7$ \\
\hline F13 & $753 \pm 12.16$ & $0.689 \pm 0.08$ & $-19.90 \pm 0.68$ & $16.73 \pm 0.2$ \\
\hline F14 & $530 \pm 14.18$ & $0.995 \pm 0.09$ & $-17.40 \pm 0.45$ & $34.54 \pm 0.05$ \\
\hline F15 & $1647 \pm 17.15$ & $0.662 \pm 0.05$ & $-18.30 \pm 0.23$ & $61.36 \pm 0.18$ \\
\hline F16 & $1592 \pm 18.19$ & $0.674 \pm 0.02$ & $-23.20 \pm 0.28$ & $66.94 \pm 0.14$ \\
\hline F17 & $2223 \pm 15.16$ & $1 \pm 0.06$ & $-25.70 \pm 0.46$ & $89.59 \pm 0.16$ \\
\hline F18 & $1672 \pm 11.89$ & $0.93 \pm 0.07$ & $-14.80 \pm 0.52$ & $92.57 \pm 0.19$ \\
\hline F19 & $246.7 \pm 10.50$ & $0.385 \pm 0.07$ & $-21.5 \pm 0.64$ & $95.06 \pm 0.25$ \\
\hline
\end{tabular}

( ${ }^{*}$ All values are expressed as mean $n=3, \pm$ SD: standard deviations)

\section{Zeta potential}

Zeta potential, which reflects the electric charge on the particles surface, was a useful parameter to predict the physical stability of colloidal systems $[14,17,32]$. The zeta potential indicated the degree of repulsion between close and similarly charged particles in the nanodispersion. The zeta potential values above $|8-9 \mathrm{mV}|$ were a prerequisite for the stability of the nanoparticles. Zeta potential less than or equal $|21 \mathrm{mV}|$ were considered as advisable for the stabilization of suspensions, since very little or no agglomeration takes place, while $|30 \mathrm{mV}|$ were indicated to full electrostatic stability [33].

The results of TFH SLNs zeta potential of different formulae are shown in table 3 . The values of zeta potential were between-10.50 $\mathrm{mV}$ and $-24.43 \mathrm{mV}$.

\section{Entrapment efficiency}

EE\% was the second dependent variable selected to study the effect of different formulation variables and their interactions. The results are listed in table 3 and illustrated in fig. 3.

\section{The effect of lipid concentration on EE \%}

The lipid concentration exerted a highly significant positive effect on EE\% of the prepared SLNs $\left({ }^{* *} \mathrm{p}<0.0001\right)$. As shown in fig. 3, a direct relationship was investigated between lipid concentration and EE\%. This was attributed either to the increase in lipids content, which may increase the space for the drug molecules to be entrapped or to the increase in the druglipid solubility, which may also lead to an increase the entrapment [12]. These results are in full agreement with (Soma 
et al., 2013), who studied the effect of increasing GMS on Irbesartan loaded SLNs. They proved that increasing concentration of GMS increased EE\% [20].

\section{The effect of stabilizer concentration on EE \%}

Changing stabilizer concentration from 1 to $2 \%$ exerted a significant effect on EE\% of the prepared SLNs $\left({ }^{*} \mathrm{p} \leq 0.05\right)$. All the SLNs formulae prepared using $2 \%$ stabilizer concentrations showed higher EE\% than their corresponding ones containing 1\% stabilizer (table 3). This was because increasing the stabilizer concentration was found to enhance the solubility of the drug and so $\mathrm{EE} \%$ was increased [26].

\section{Effect of Stabilizer type on EE\%}

Studying the effect of stabilizer's type on the EE\% showed that Tween 80 affected the EE\% significantly $\left({ }^{*} \mathrm{p}<0.05\right)$, while both Cremophor RH40 and Poloxamer188 exerted a non-significant effect on it ( $\mathrm{p}$ values $>0.05$ ). This may be due to the difference in chemistry of these stabilizers, which may affect the solubility of the drug in lipid either by increasing or decreasing it, and this will affect EE\%.

These results of studying the effect of changing both the type and concentration of stabilizer are in agreement with Ekambaram and Abdul Hasan Sathali, who reported that increasing the concentration of stabilizers from $1 \%$ to $2 \%$ affected EE\% irrespective of the type of stabilizer [26].
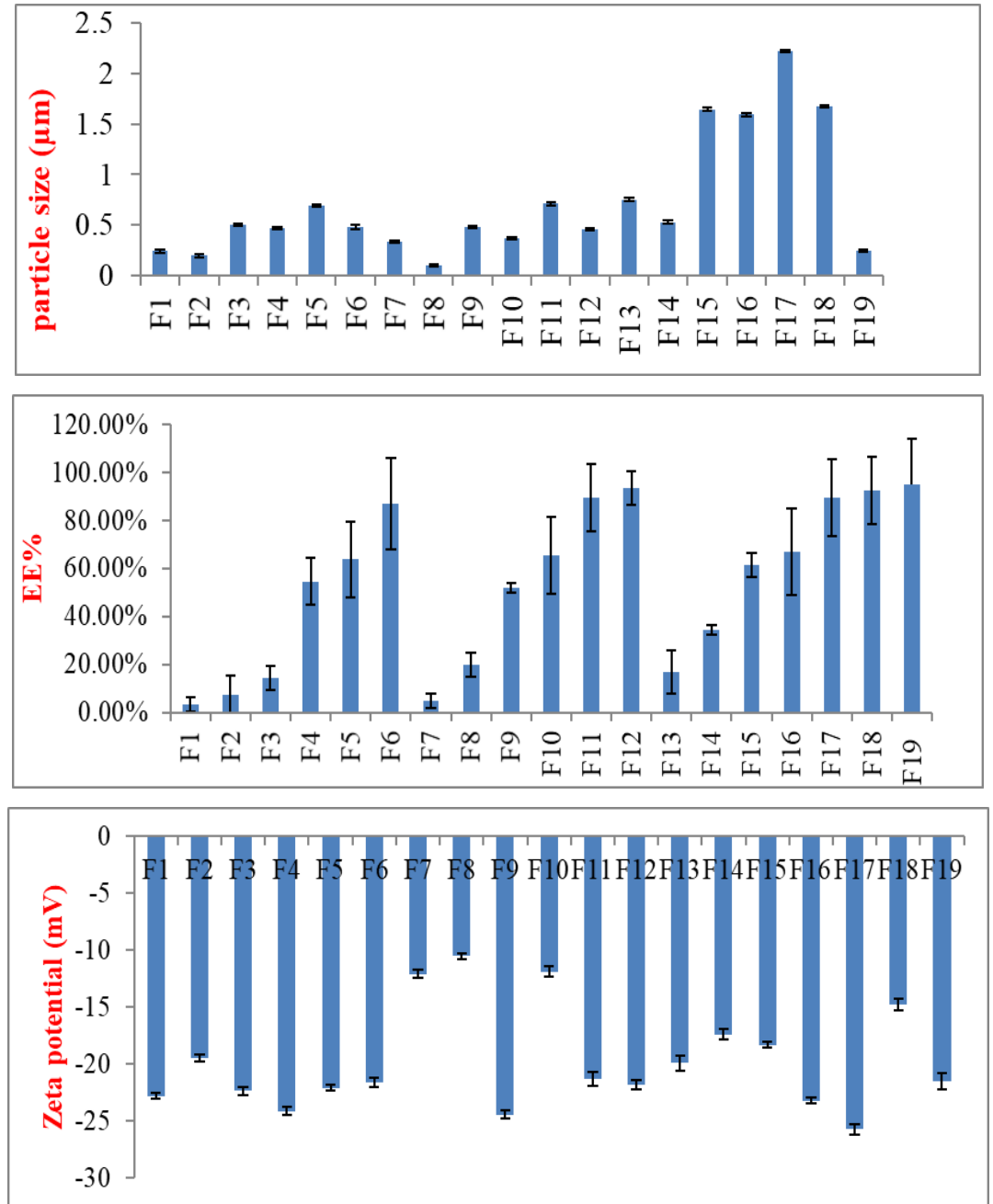

Fig. 3: Histogram of particle size, entrapment efficiency, and zeta potential of TFH loaded SLNs, $(n=3 \pm$ SD)

\section{Selection of the optimized formulae}

After a complete analysis of the factorial design with the previously set levels of the selected independent variables and their effect on the selected important dependant variable of particle size and EE\%, the two optimum criteria set were suitable particle size and highest EE\%. Suitable PDI was also taken into consideration. According to these criteria, the three optimum formulae (F6, F12, and F19) were selected for further evaluation.

\section{Morphology of prepared SLN formulae}

Optimized formulae were selected for studying their surface morphology as they show suitable particle size with the best EE\%. The morphology of fresh TFH SLNs was observed using TEM. Morphology TEM was shown in fig. 4 for F6, F12, and F19.
TEM photographs revealed that the particles possessed a nanometer-size with spherical uniform shape without aggregation. The particle size ranged from 480 to $490 \mathrm{~nm}$ for F6, from 455 to 470 $\mathrm{nm}$ for F12 and from 245 to $260 \mathrm{~nm}$ for F19.

\section{In vitro drug release study}

The drug was released from SLNs and diffused through the cellulose nitrate membrane into the release medium. Drug release was measured by determining the drug concentration in the release medium. Fig. 5 illustrates the release profile of TFH from optimized SLNs. The cumulative release profiles were constructed up to 150 min. For assessment and comparison, the release profiles evaluation was based on the cumulative drug \% release and T50\%. A nonsignificant difference was recorded when comparing the cumulative drug release from the formulae $(P 0.05)$. The cumulative drug 
release was $74.58 \%, 70.56 \%$ and $66.91 \%$ for formulae F6, F12 and F19, respectively. In order to determine the release model, the in vitro drug release data were analyzed according to zero order, first order, and diffusion-controlled mechanism according to Higuchi model. The results revealed that the release of TFH from all the three optimized formulae obeyed the Higuchi model of matrix diffusion with highest $\mathrm{R}^{2}$ values, as shown in table 4 . This is in agreement with Gardouh et al. who stated that drug release by diffusion involves three steps which include penetration of water into system causing swelling of matrix followed by the conversion of solid lipid into rubbery matrix, and then the diffusion of drug from the swollen rubbery matrix takes place [34]. To confirm the release mechanism, the data were fitted to the Korsmeyer-Peppas equation to describe the drug release mechanism from matrix. It was revealed that the three formulae showed non-Fickian diffusion with $\mathrm{n}$ values $0.45<\mathrm{n}<0.89$. T50\% recorded a value of $49.22 \pm 1.6,59.46 \pm 0.7$ and $61.45 \pm 1.3$ min for F6, F12 and F19, respectively. They showed a nonsignificant difference. $(\mathrm{P}>0.05)$.
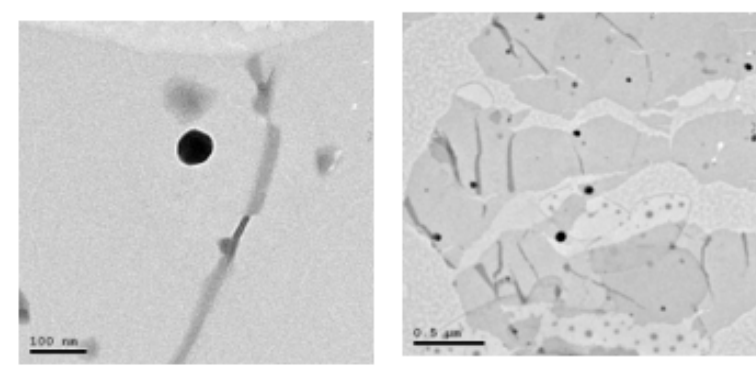

F6
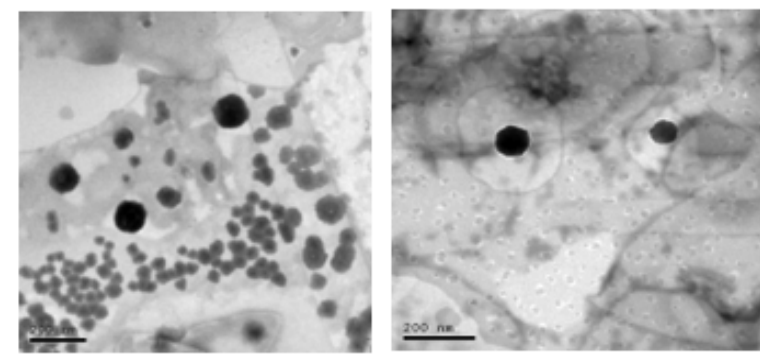

F12
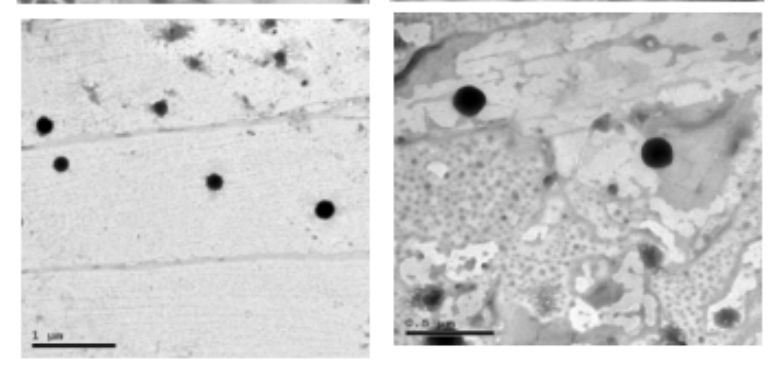

Fig. 4: Transmission electron microscope photographs of the selected formulae, $(n=3 \pm S D)$

Table 4: In vitro drug release kinetics of TFH from SLN formulae

\begin{tabular}{llllll}
\hline $\begin{array}{l}\text { Formula } \\
\text { code }\end{array}$ & $\begin{array}{l}\text { Zero- } \\
\text { order }\end{array}$ & $\begin{array}{l}\text { First- } \\
\text { order }\end{array}$ & $\begin{array}{l}\text { Higuchi diffusion } \\
\text { model }\end{array}$ & $\begin{array}{l}\text { Korsmeyer-Peppas } \\
\text { model }\end{array}$ & $\begin{array}{l}\text { n-korsmeyer- } \\
\text { peppas model }\end{array}$ \\
\hline & $\mathrm{R}^{2}$ & $\mathrm{R}^{2}$ & $\mathrm{R}^{2}$ & $\mathrm{R}^{2}$ & $\mathrm{n}$ \\
$\mathrm{F} 6$ & 0.942 & 0.820 & 0.987 & 0.811 & 0.866 \\
F12 & 0.859 & 0.74 & 0.942 & 0.877 & 0.848 \\
F19 & 0.937 & 0.763 & 0.978 & 0.0 .781 & 0.814 \\
\hline
\end{tabular}

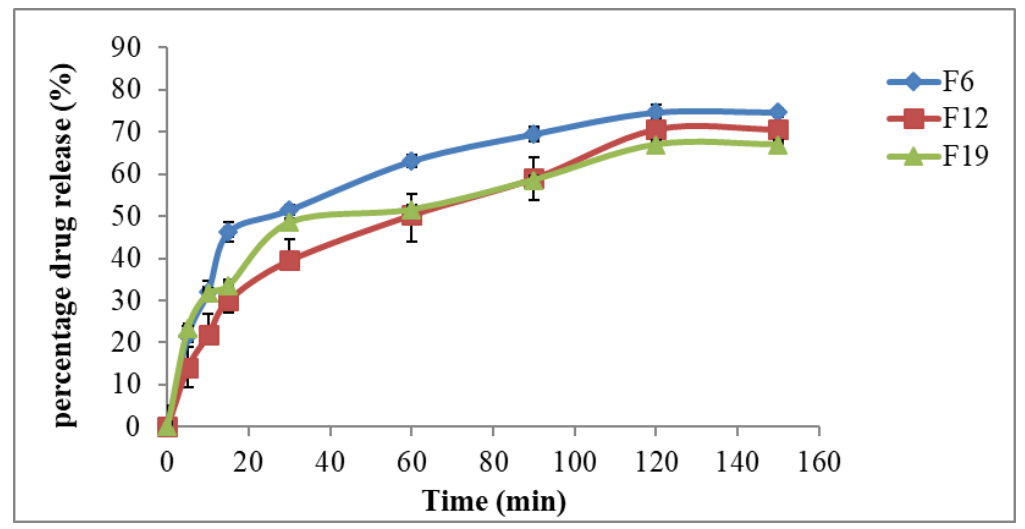

Fig. 5: Cumulative release profiles of TFH loaded SLNs, $(n=3 \pm S D)$ 


\section{Infrared (IR) spectroscopy}

FT-IR spectroscopy was used to characterize possible interactions between the drug and the used excipients. The interactions between them often lead to identifiable changes in the IR profile of the formulae. FT-IR spectra of TFH, TFH/Tween 80 physical mixture (1:1), TFH/GMS physical mixture (1:1), TFH/Cremophor RH40 physical mixture (1:1), F6, F12 and F19were shown in fig. (6a, b,c,d, e, fand g). TFH had characteristic IR peaks at 3038 (alkenyl C-H stretch and/or aromatic C-H stretch), 2970 (alkyl C-H stretch), 2,225 (alkynyl €C stretch), 1,596 (alkenyl C0C stretch), 1,490 (aromatic C0Cbending) $\mathrm{cm}^{-1}$ [35]. The IR spectrum of the developed physical mixtures, as well as the formulae (F6, F12, and F19), showed minor differences in the positions of the absorption bands of TFH. These indicated the incorporation of the drug in SLNs formulae without any interaction with neither the lipid nor the stabilizers.

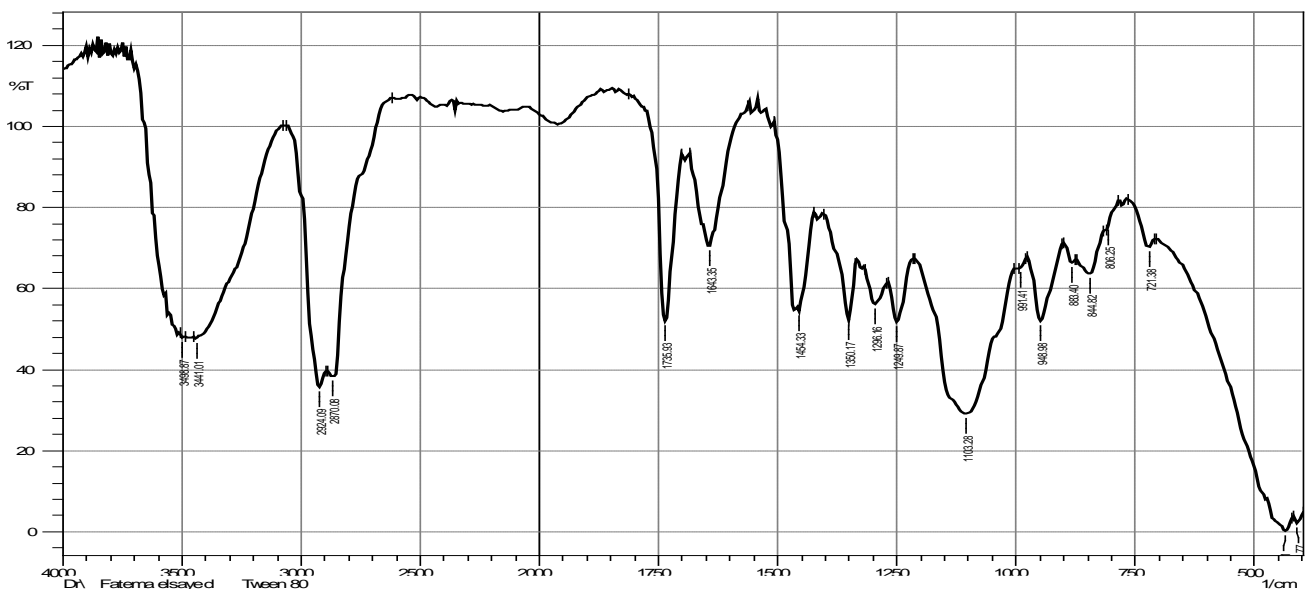

(a)

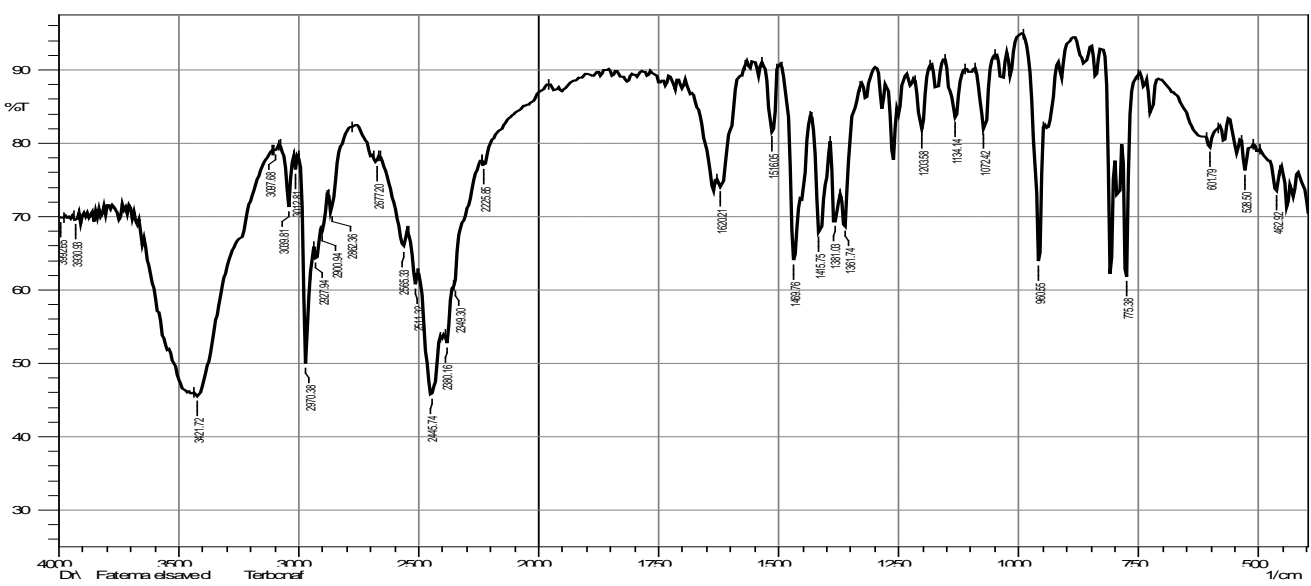

(b)

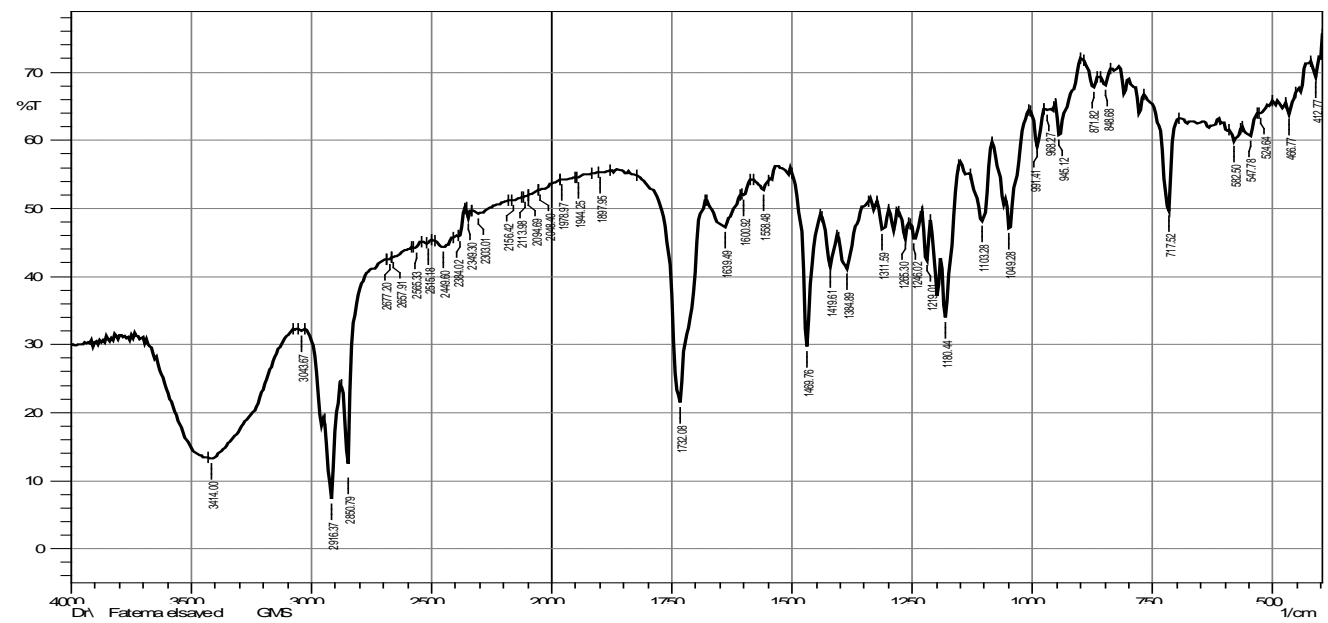

(c) 


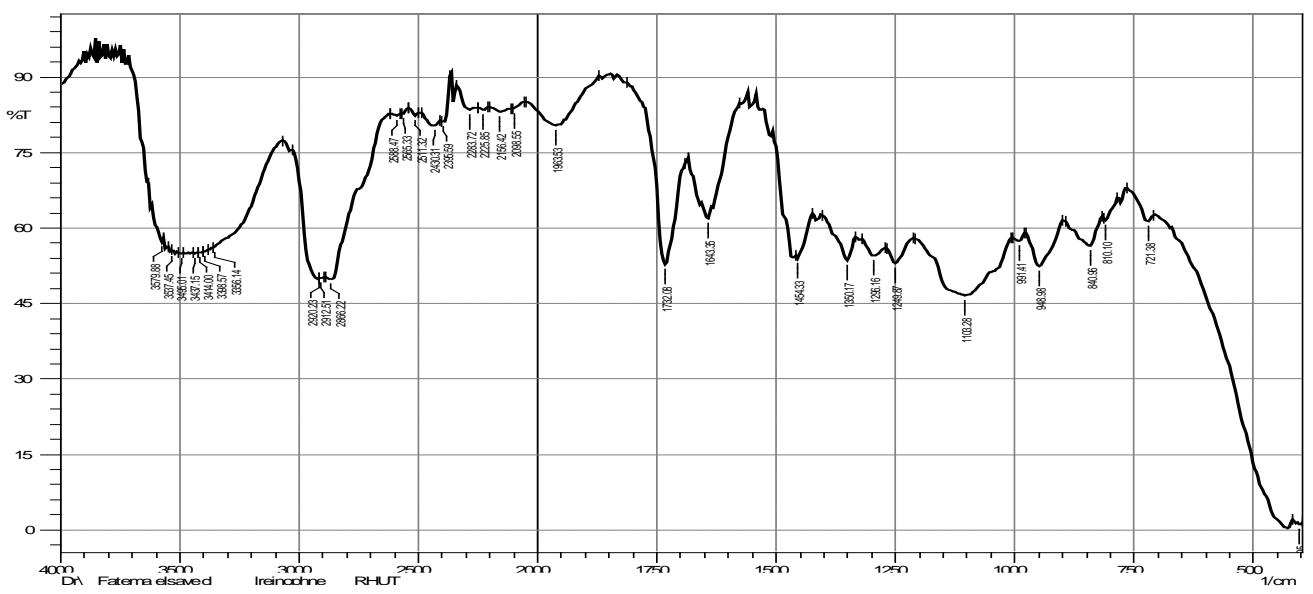

(d)

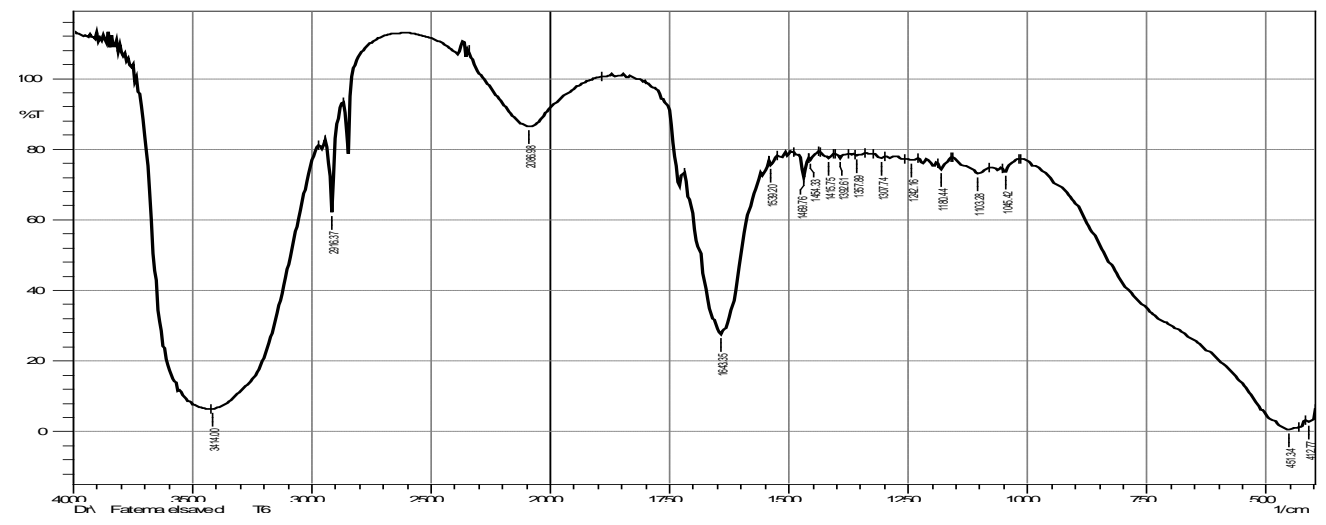

(e)

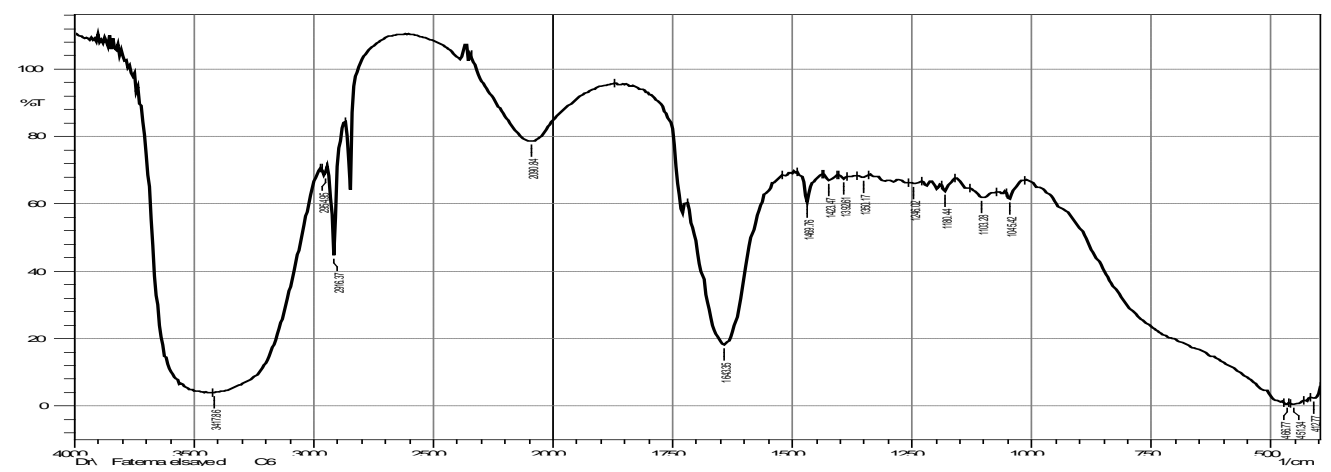

(f)

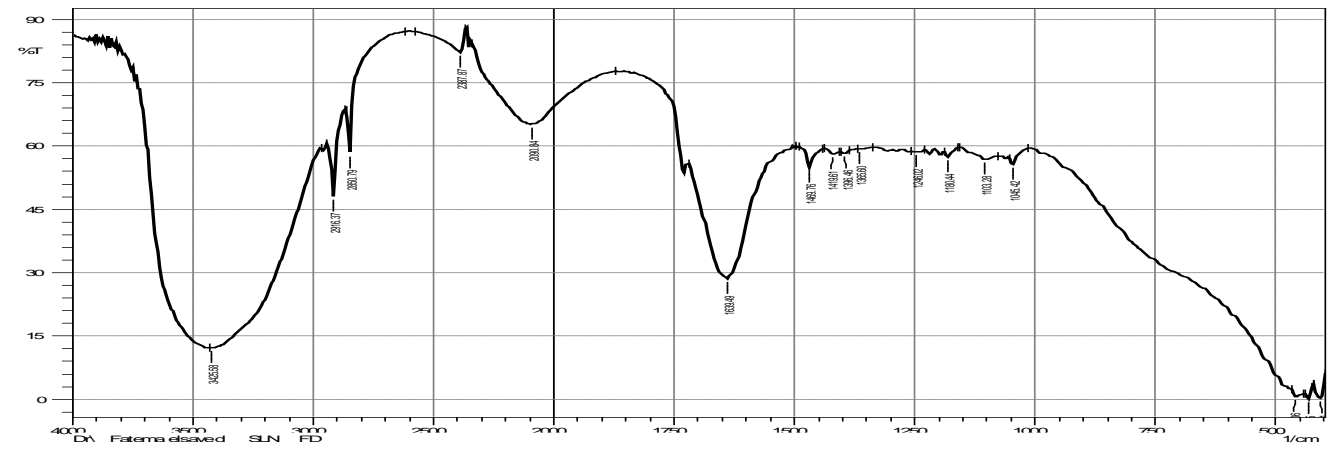

(g)

Fig. 6: FT-IR spectra of TFH (a), TFH/Tween 80 physical mixture (1:1) (b), TFH/GMS physical mixture (1:1) (c), TFH/Cremophor RH40 physical mixture (1:1) (d), FT-IR spectra of F6 (e), FT-IR spectra of F12 (f) and FT-IR spectra of F19 (g) 


\section{In vitro microbiology assessment}

This test was used to prove the efficacy of the selected SLNs formulae against fungal infection caused by Candida albicans. Fig. 7 show the diameters of zones of inhibition $(\mathrm{mm})$ caused by the selected SLNs formulae, plain base for each formula, and the marketed lamifen ${ }^{\circledR}$ cream. The larger the zone of inhibition of
Candida albicans growth, the higher the release rate of the drug from the SLNs and the greater the efficacy of the released drug towards killing the Candida albicans. Formulae F6, F12 and F19, showed a mean zone of inhibition of $25.9 \pm 0.25 \mathrm{~mm}, 25 \pm 0.35 \mathrm{~mm}$ and $24.67 \pm 0.36 \mathrm{~mm}$, respectively, which were significantly greater than the mean zone of inhibition exhibited by the commercial preparation lamifen ${ }^{\circledR}$ cream $(11.2 \pm 0.44 \mathrm{~mm})\left({ }^{*} \mathrm{P}<0.05\right)$.
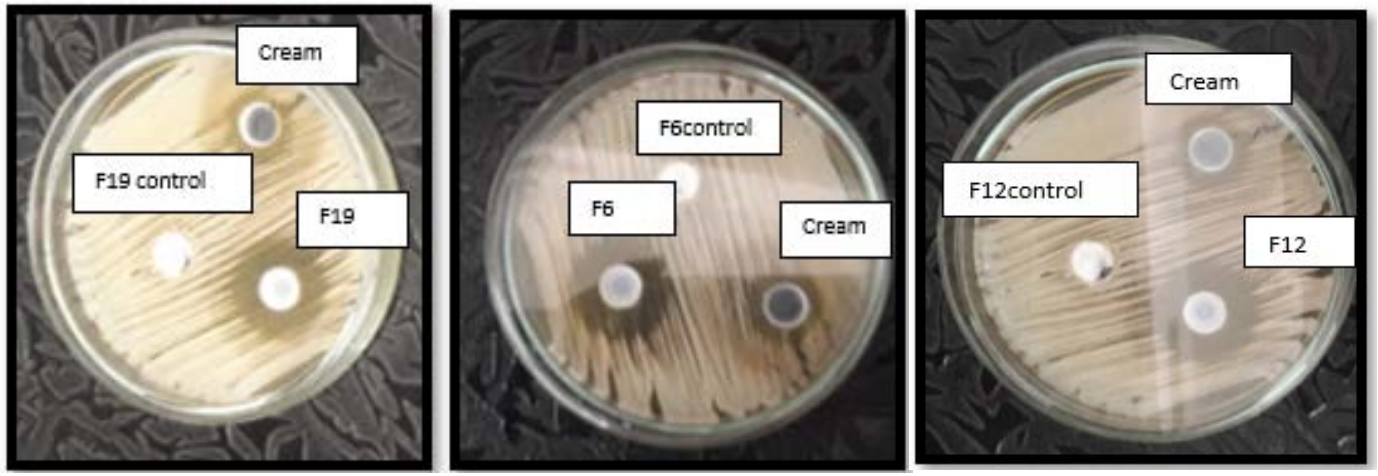

Fig. 7: Microbiological agar plate showing zones of inhibition induced by F6, F12, and F19 formulae, plain formula (control), and the marketed product, $(n=3 \pm S D)$

\section{Effect of storage time on the stability of the selected formulae}

The optimized SLNs formulae were stored under ambient conditions for 3 and 6 mo at $25{ }^{\circ} \mathrm{C}$. They were re-evaluated for their particle size and $\mathrm{EE} \%$. A slight increase in the particle size diameter (fig. 8) and slight decrease of EE\% (fig. 9) were observed; both were statistically non-significant $(\mathrm{P}>0.05$, paired t-test), proving the stability of TFH loaded SLNs.

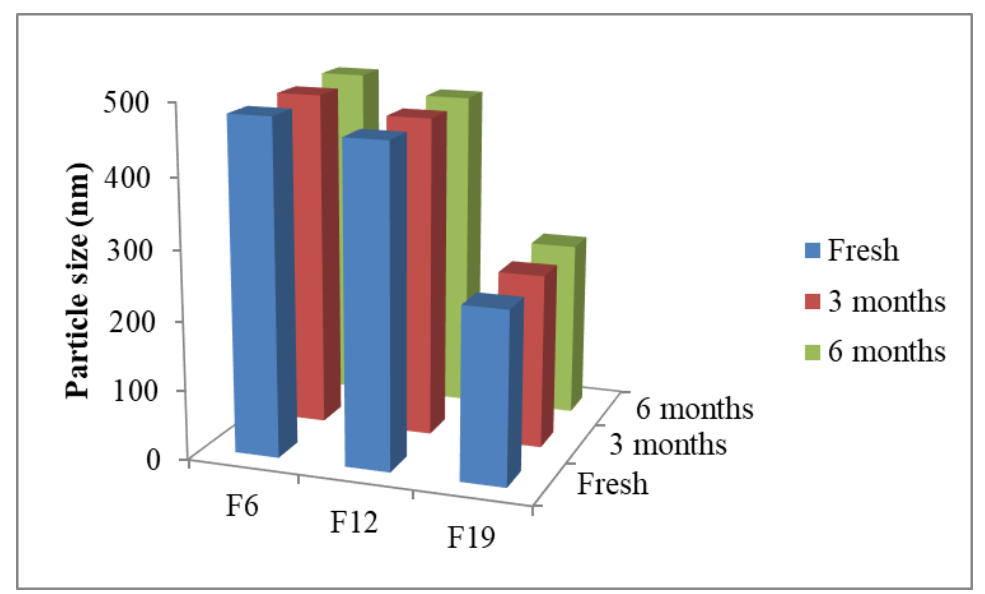

Fig. 8: Effect of storage on the particle size of SLNs after three and six months at $25^{\circ} \mathrm{C},(\mathrm{n}=3 \pm \mathrm{SD})$

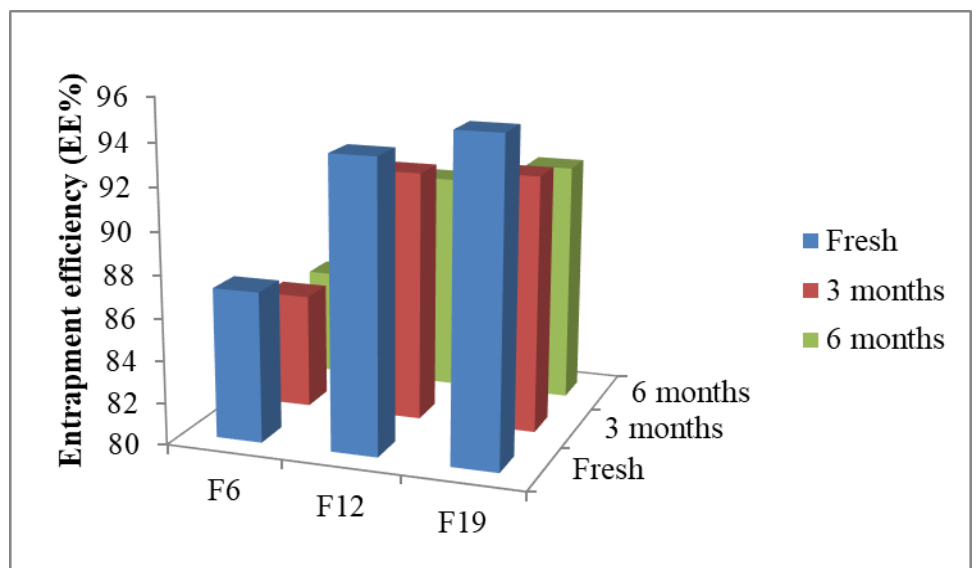

Fig. 9: Effect of storage on the EE\% of SLNs after three and six months at $25^{\circ} \mathrm{C},(n=3 \pm S D)$ 


\section{CONCLUSION}

In this design, it was noted that lipid concentration exerted a significant effect on particle size and EE\%. The type of stabilizer chosen and its concentration also showed a significant effect on the studied parameters. The selected optimized formulae of the prepared TFH SLNs (F6, F12, and F19) obtained optimum particle size with the highest EE\%. They showed satisfactory in vitro release profiles. In vitro microbiology testing depicted that the zone of inhibition of these three formulae was visibly more than that observed from the market cream (lamifen ${ }^{\circledR}$ cream).

\section{ACKNOWLEDGMENT}

We are thankful to Amoun Pharmaceutical Company for providing a sample of drug and excipients. We are very grateful to dr Walaa $\mathrm{Al}$ Shareef, lecturer of microbiology, Faculty of Pharmacy, October $6^{\text {th }}$ University, for helping us in performing the microbiological studies. Great thanks to our organization (NODCAR) and Faculty of Pharmacy October $6^{\text {th }}$ University for providing their laboratories and facilities to complete the research work.

\section{AUTHORS CONTRIBUTIONS}

The research idea was set with guidance of Prof. Dr. Wedad Sakran and Dr. Vivian S. Elwazzan. The research work and manuscript preparation were done by Fatma E. Abobakr; the research work was guided by Dr. Sahar M. Fayez, Calculations and data interpretation was supported by Dr. Vivian S. Elwazzan, critical revision of the manuscript was done by Dr. Sahar M. Fayez, and Dr Vivian S. Elwazzan and the final version of the manuscript were approved by Prof. Dr. Wedad. Sakran.

\section{CONFLICTS OF INTERESTS}

\section{Declared none}

\section{REFERENCES}

1. Westesen K. Novel lipid-based colloidal dispersions as potential drug administration systems-expectations and reality. Colloid Polym Sci 2000;278:608-19.

2. Remya PN, Damodharan N. Formulation, development, and characterisation of cilnidipine loaded solid lipid nanoparticles. Asian J Pharm Clin Res 2018;11:120-5.

3. Bhoskar M, Patil P. Development and evaluation of paclitaxel loaded nanoparticles using 24 factorial design. Int J Current Pharm 2015;7:64-72.

4. Reis C, Neufeld R, Ribeiro A, Veiga F. Nanoencapsulation I methods for preparation of drug-loaded polymeric nanoparticles. Nanomed Nanotechnol 2006;1:8-21.

5. Shah R, Eldridge D, Palombo EHI. Composition, and structure in lipid nanoparticles: production, characterization, and stability. chapter I (Introduction); 2015. p. 2-3.

6. Raut ID, Manjappa AS, Mohite SK, Doijad RC. Design,development and characterization of paclitaxel loaded solid lipid nanoparticles as a colloidal drug carrier. Int J Appl Pharm 2019;11:333-40.

7. Corrigan 0, Healy A. Surfactants in pharmaceutical products and systems. In: Swarbrick J. (ed) Encyclopedia of pharmaceutical technology. 3rd edn. Informa Healthcare, NY, USA; 2006. p. 3583-96.

8. Kanakapura B, Penmatsa VK. Analytical methods for determination of terbinafine hydrochloride in pharmaceuticals and biological materials. J Pharm Anal 2016;6:137-49.

9. Gaba B, Fazil M, Khan S, Ali A, Baboota S, Ali J. Nanostructured lipid carrier system for topical delivery of terbinafine hydrochloride. Bull Fac Pharm 2015;53:147-59.

10. Schubert MA, Muller Goymann CC. Solvent injection as a new approach for manufacturing lipid nanoparticles evaluation of the method and process parameters. Eur J Pharm Biopharm 2003;1:125-31.

11. Parhi R, Suresh P. Production of solid lipid nanoparticlesdrug loading and release mechanism. J Chem Pharm Res 2010;2:211-27.

12. Mora Huertasa CE, Fessi H, Elaissari A. Polymer-based nanocapsules for drug delivery. Int J Pharm 2010;385:113-42.

13. Amin MM, Sheta NM, Abd El, Gawad NA, Badawi AA. Preparation and characterization of benzophenone-3 loaded polymeric nanoparticles of lactide-co-caprolactone as drug carriers. J Pharm Res Opinion 2012;2:28-41.

14. Chandran SP, Nachimuthu K. Formulation and characterization of papain loaded solid lipid nanoparticles against human colorectal adenocarcinoma cell line. Asian J Pharm Clin Res 2018;11:393-9.

15. Vitorino C, Carvalho FA, Almeida AJ, Sousa JJ, Pais A. The size of solid lipid nanoparticles: an interpretation from experimental design. Colloids Surfaces B: Biointerfaces 2011;84:117-30.

16. Patel KK, Marya BH, Kakhanis VV. Spectrophotometric determination and validation for terbinafine hydrochloride in pure and in tablet dosage form. Scholars Research Library 2012;4:1119-22.

17. Ramesh Reddy M, Saty Anarayana SV, Jayasankar Reddy V. Development and evaluation of clobetasol-loaded solid lipid nanoparticles for topical treatment of psoriasis. Int J Appl Pharm 2019;11:143-50.

18. Montenegro L, Carbone C, Puglisi G. Vehicle effects on in vitro release and skin permeation of octylmethoxycinnamate from microemulsions. Int J Pharm 2011;405:162-8.

19. USPharmacopeia 42 and National Formulary 37; 2019;2:4233.

20. Soma D, Attari Z, Reddy MS, Damodaram A. Solid lipid nanoparticles of irbesartan: preparation, characterization, optimization, and pharmacokinetic studies. Braz J Pharm Sci 2017;1:1-10.

21. Barot BS, Parejiya PB, Patel HK, Gohel MC, Shelat PK. Microemulsion-based gel of terbinafine for the treatment of onychomycosis: optimization of formulation using D-optimal design. AAPS PharmSciTech 2012;13:184-92.

22. Sahoo S, Pani NR, Sahoo SK. Effect of microemulsion in topical sertaconazole hydrogel: in vitro and in vivo study. Drug Delivery 2014;20:1-8.

23. Devi M, Kumar SM, Mahadevan N. Amphotericin-B loaded vesicular systems for the treatment of topical fungal infection Int J Res Adv Pharm Res 2011;4:37-46.

24. Shahin M, Abdel Hady S, Hammad M, Mortada N. Novel jojoba oil-based emulsion gel formulations for clotrimazole delivery. AAPS PharmSciTech 2011;12:239-47.

25. Verma S, Bhardwaj A, Vij M, Bajpai P, Goutam N, Kumar L. Oleic acid vesicles: a new approach for topical delivery of antifungal agent. Artif Cells Nanomed Biotechnol 2014;42:95-101.

26. Ekambaram P, Abdul Hasan Sathali A. Formulation and evaluation of solid lipid nanoparticles of ramipril. J Young Pharm 2011;3:216-20.

27. Priyanka K, Abdul Hasan SA. Preparation and evaluation of montelukast sodium loaded solid lipid nanoparticles. J Young Pharm 2012;4:129-37.

28. Tiwari S, Mistry P, Patel V. SLNs based on co-processed lipids for topical delivery of terbinafine hydrochloride. J Pharm Drug Dev 2014;1:1-8.

29. Diniyanti SN, Werawatganone P, Muangsiri W. Preparation of solid lipid nanoparticles containing mangosteen pericarp extract. Asian J Pharm Clin Res 2018;11:80-4.

30. Shahi M, Pathak. Development and statistical optimization of solid lipid nanoparticles of simvastatin by using $2^{3}$ full-factorial design. AAPS PharmSciTech 2010;2:489-96.

31. Leroux JC, Allemann E, Doelker E, Gurny R. New approach for the preparation of nanoparticles by an emulsification-diffusion method. Eur J Pharm Biopharm 1994;41:14-8.

32. Khalil RM, El-bary AA, Kassem MA, Ghorab MM, Ahmed MB. Solid lipid nanoparticles for topical delivery of meloxicam: development and in vitro characterization. $1^{\text {st }}$ Annual International Interdisciplinary Conference; 2013. p. 24-6.

33. Hernandez JRV, Goymann CCM. Novel nanoparticulate carrier system based on carnauba wax and decyl oleate for the dispersion of inorganic sunscreens in aqueous media. Eur J Pharm Biopharm 2005;60:113-22.

34. R Gardouh, Shadeed Gad, Hassan M Ghnaim, Mamdouh M. Ghorab design and characterization of glyceryl monostearate solid lipid nanoparticles prepared by high shear homogenization. BJPR 2013;3:326-46.

35. Tayel SA, El-Nabarawi MA, Tadros MI, Abd-elsalam WH. Positively charged polymeric nanoparticle reservoirs of terbinafine hydrochloride: preclinical implications for controlled drug delivery in the aqueous humor of rabbits. AAPS PharmaSciTech 2013;14:782-93. 